\title{
Stage III Duodenal Neuroendocrine Tumor AJCC v8
}

National Cancer Institute

\section{Source}

National Cancer Institute. Stage /II Duodenal Neuroendocrine Tumor A/CC v8. NCI

Thesaurus. Code C135078.

Stage III includes: (T4, N0, M0); (Any T, N1, M0). T4: Tumor invading the visceral peritoneum (serosa) or other organs. N0: No regional lymph node metastasis. N1: Regional lymph node metastasis. M0: No distant metastasis. (AJCC 8th ed.) 